\title{
Grupos de riesgo para tuberculosis en Chile
}

\author{
Tania Herrera
}

\section{Risk groups for tuberculosis in Chile}

Tuberculosis (TB) remains a major global and national problem. In Chile the incidence rate has remained at 13 per 100,000 inhabitants for several years without tendency to the expected decline that would allow their elimination by 2020 . As a low prevalence country, TB cases have been concentrated in risk groups, reaching $33 \%$ in 2013 , and this proportion increases as younger people are analyzed. The main risk groups in Chile are HIV co-infection, foreigners and population of prisons. By 2013, the proportion of cases for these three groups was $8.7 \%, 8.4 \%$ and $3.9 \%$ respectively, and these percentages vary significantly when regional situation is analyzed. In addition, many of these patients have more than one risk factor, demonstrating the existence of clusters more vulnerable to TB.

Key words: Tuberculosis, epidemiology, risk groups, control.

Palabras clave: Tuberculosis, epidemiología, grupos de riesgo, control.

\section{Introducción}

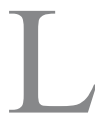

a tuberculosis $(\mathrm{TBC})$ corresponde a la segunda causa de muerte por una enfermedad infecciosa en el mundo, después del virus de la inmunodeficiencia humana (VIH) y continúa siendo un problema de gran magnitud a escala mundial. Para el año 2013, la Organización Mundial de la Salud reportó 9 millones de casos nuevos y 1,5 millones de personas fallecidas por esta causa $^{1}$. En Chile, a pesar de que la incidencia es menor que en la mayoría de los países de Latinoamérica, la TBC continúa siendo una enfermedad presente, produciéndose 13 casos nuevos por cada 100.000 habitantes cada año y sin observarse la velocidad de disminución que se requeriría para lograr su eliminación al año $2020^{2}$.

Las causas de este estancamiento en las tasas de incidencia de $\mathrm{TBC}$ en Chile son múltiples y se repiten en otros países con situación epidemiológica similar. Los esfuerzos que el sistema de salud debe desarrollar para diagnosticar y tratar los casos son mayores y se requiere de la participación de otros programas de salud y del intersector debido a que la población afectada se va haciendo cada vez más compleja ${ }^{3,4}$. Sin embargo, la realidad de los países muestra que los recursos asignados al programa van disminuyendo en la medida que el problema pareciera menor en magnitud, lo que produce su debilitamiento y con ello la mantención de las fuentes de contagio y la perpetuación de la endemia 5 .

Una de las estrategias propuestas para los países de baja prevalencia es mejorar la eficiencia de la pesquisa de la TBC pulmonar bacilífera focalizando las acciones en los llamados grupos de riesgo para $\mathrm{TBC}$, los que corresponden a personas que por factores biológicos o socio-económicos son más vulnerables a desarrollar la enfermedad ${ }^{4,6,7}$. El Programa Nacional de Control y Eliminación de la Tuberculosis (PROCET) identifica una serie de grupos poblacionales que tienen un riesgo mayor de enfermar de TBC: contactos de pacientes con TBC pulmonar confirmada por bacteriología, co-infectados con VIH, población privada de libertad, adultos mayores, personas pertenecientes a pueblos indígenas, personas en situación de calle, extranjeros provenientes de países de alta endemia, residentes de instituciones cerradas, diabéticos o personas con otras inmunosupresiones, alcoholismo/ drogadicción y el personal de salud ${ }^{8}$.

El presente artículo expone la situación actual de la TBC en los principales grupos de riesgo en Chile, cuantificando el aporte que cada uno hace al total de casos de TBC en el país e identificando algunas características demográficas y de distribución territorial que permitan contribuir al diseño de estrategias de abordaje para el diagnóstico precoz de la enfermedad.

\section{Grupos de riesgo}

La vigilancia específica de la TBC en Chile incluye, además de la notificación de la enfermedad y la vigilancia de laboratorio, la entrega de la información mensual de los casos en tratamiento por parte de los establecimientos de salud. En este registro se debe dar cuenta de la pertenencia de cada paciente a los grupos de riesgo especificados en el Programa ${ }^{8}$. En los últimos años, el porcentaje de los casos de TBC que pertenecía a algún grupo de riesgo, excluyendo el hecho de ser adulto mayor, ha ido aumentando, siendo de $20 \%$ el $2011,23 \%$ el 2012 y $33 \%$ el $2013^{2}$.
Ministerio de Salud. Chile. Directora del Programa de Control y Eliminación de la Tuberculosis.

Artículo por invitación. Recibido: 30 de diciembre de 2013

Correspondencia a: Tania Herrera Martínez Tania.herrera@minsal.cl 
Proporcionalmente, los principales grupos de riesgo reportados en Chile, además de los adultos mayores que constituyen $20 \%$ de los casos, son los pacientes con co-infección por $\mathrm{VIH}$, los inmigrantes, personas que presentan abuso de sustancias (alcoholismo y drogadicción), las personas privadas de libertad, los pueblos indígenas y las personas en situación de calle ${ }^{2}$. La Tabla 1 muestra la proporción de casos para estos grupos durante el año 2013. Cabe señalar que una misma persona puede tener más de un factor de riesgo, por lo que la suma del aporte de cada uno no da cuenta del total. Ahora, si se consideran las tasas de incidencia estimadas específicas para estos grupos, los más importantes son los contactos, los pacientes con co-infección VIH, las personas extranjeras y las personas privadas de libertad ${ }^{7}$.

Mención especial se debe hacer a la diabetes mellitus ya que, a pesar de presentar un bajo reporte, estudios en la Región Metropolitana muestran su presencia en 15\% de los casos, por lo que el sub-registro es evidente?

Por otra parte, para el grupo de 15 a 64 años, el porcentaje de pacientes que pertenecen a un grupo de riesgo es de $39 \%$ ( $45 \%$ en los hombres y $28 \%$ en mujeres). La co-infección con VIH llega a 10\%, los extranjeros también son $10 \%$, las personas privadas de libertad son el 5,1\% y la presencia de alcoholismo o drogadicción se encuentra en $6,1 \%$.

En el grupo de pacientes entre los 15 y 44 años, el porcentaje que pertenece a un grupo de riesgo en mayor aún, llegando a $45 \%$ ( $53 \%$ en hombres y $32 \%$ en mujeres). En ellos la co-infección con VIH alcanza a 13\%, los extranjeros son el $16 \%$, las personas privadas de libertad son $7,2 \%$ y la presencia de alcoholismo o drogadicción se encuentra en 5,4\%. Se observa, entonces, que a excepción del abuso de sustancias, los principales factores de riesgo para TBC van aumentando su presencia a medida que las personas que se enferman son más jóvenes, lo que refuerza

Tabla 1. Proporción de casos de tuberculosis -todas las formas- según grupos de riesgo. Chile 2013

\begin{tabular}{lc}
\hline Grupo de riesgo & $\%$ \\
Co-infección VIH & $8,7 \%$ \\
Extranjeros & $8,4 \%$ \\
Alcoholismo/drogadicción & $6,4 \%$ \\
Población privada de libertad & $3,9 \%$ \\
Diabetes mellitus & $3,4 \%$ \\
Pueblo indígena & $2,5 \%$ \\
Situación de calle* & $2,4 \%$ \\
Contactos & $1,4 \%$
\end{tabular}

Fuente: Programa de Tuberculosis. Informe de situación 2013 *Sinónimo: vagabundo. la necesidad de focalizar los esfuerzos de pesquisa en estos grupos.

Finalmente, en el grupo bajo 15 años, 30\% registra un factor de riesgo, siendo el más importante el antecedente de contacto $(16 \%)$, seguido de pertenecer a un pueblo indígena (7\%) y ser extranjero (5\%). En todo caso, en los pacientes bajo 15 años se esperaría que la mayoría tuviera el antecedente de ser contacto, por lo que la probabilidad de sub-registro es alta.

A continuación se describe la situación específica para la co-infección con VIH, los extranjeros y las personas privadas de libertad.

\section{Co-infección con VIH}

La co-infección TBC-VIH como factor de riesgo para desarrollar TBC es ampliamente conocida. La OMS señala que mundialmente, $13 \%$ del total de casos de TBC se presenta en personas infectadas por el VIH, siendo causa de 320.000 muertes en $2012^{10}$. Estudios realizados en Chile estiman una prevalencia de TBC en pacientes con VIH 300 veces superior a la de la población general, constituyendo uno de los principales grupos de riesgo ${ }^{11}$.

Para el año 2013, 8,7\% de los casos de TBC se presentaron en personas con infección por $\mathrm{VIH}$, proporción que varía dependiendo de la región. Así, las regiones que presentan sobre $10 \%$ de co-infección fueron Arica y Parinacota, Antofagasta, Atacama, Coquimbo y Magallanes.

El 90\% de los pacientes con TBC-VIH son hombres y el promedio de edad es de 38 años (rango 16 a 69 años). El 51\% presentó TBC pulmonar confirmada por bacteriología (baciloscopia, cultivo o reacción de polimerasa en cadena-RPC) y $29 \%$ presentó tuberculosis extra pulmonar. E1 87\% de los casos del año 2013 fueron casos nuevos y $15 \%$ presentó otro factor de riesgo además del VIH (privación de libertad, extranjero, alcoholismo/drogadicción y situación de calle).

\section{Migrantes}

Los datos reportados al Programa de Tuberculosis corresponden a la nacionalidad del paciente, lo que no necesariamente corresponde al término "migrante", partiendo de la base que no existe una definición internacional universalmente aceptada del término ${ }^{12}$. En concreto, puede tratarse desde personas que están en tránsito y a quienes se les diagnostica la enfermedad en Chile hasta personas asentadas en el país hace muchos años. En el año 2013, se reportaron 193 pacientes de nacionalidad distinta a la chilena, constituyendo $8 \%$ del total de morbilidad (casos nuevos y recaídas) y $8,4 \%$ de la incidencia. La distribución por nacionalidad se observa en la Figura 1, dando cuenta de que más de la mitad de los casos corresponde a personas de nacionalidad peruana, seguida de la boliviana. Además, en un alto porcentaje de casos se desconoce la nacionalidad. 
La distribución de los casos de TBC en extranjeros es muy heterogénea en el país. Así, 98\% de los casos corresponden a pacientes residentes en cuatro regiones: Metropolitana (67\%), Iquique (16\%), Antofagasta (10\%) y Arica y Parinacota (5\%). En la Región Metropolitana, los casos se concentran en los Servicios de Salud Metropolitano Norte (41\%) y Central (38\%).

El aporte de los casos de TBC en extranjeros a los casos de estos Servicios de Salud se muestra en la Tabla 2.

Entre las características demográficas de los pacientes extranjeros, $54 \%$ son hombres y el promedio de edad es de 31 años (rango de 11 a 80 años). El 68\% presentó TBC pulmonar confirmada por bacteriología (baciloscopia, cultivo o RPC) y 19\% presentó TBC extra pulmonar. El $96 \%$ de los casos del 2013 fueron casos nuevos y 12\% presentó otro factor de riesgo además de la nacionalidad no chilena, siendo los principales infección por VIH $(3,6 \%)$, pueblo indígena $(3,1 \%)$ y privación de libertad $(2,6 \%)$.

\section{Personas privadas de libertad}

Se estima que el riesgo de enfermar de TBC en las cárceles es cuatro a cinco veces mayor que en la población general. Estudios en Chile muestran un riesgo entre 15 y 18 veces mayor que en la comunidad, siendo los principales determinantes de este riesgo las determinantes sociales de estas personas, los altos niveles de hacinamiento en las cárceles y el deficiente acceso a la atención de salud ${ }^{7,13}$. En el año 2013 se reportaron 94 casos de TBC en reos, 40\% de los cuales pertenecía a la Región Metropolitana, siendo el Servicio de Salud Metropolitano Central (Penitenciaría de Santiago) el que contribuyó con 55\% de ellos.

La proporción de casos de TBC en privados de libertad es de 3,9\% para el país, pero llegó a $14 \%$ en el Servicio Metropolitano Central, $12 \%$ en Atacama y $11 \%$ en Chiloé.

El $96 \%$ de estos casos se produjo en hombres. El promedio de edad es de 34 años (rango 18 a 63 años). El $77 \%$ presentó TBC pulmonar confirmada por bacteriología (baciloscopia o cultivo) y 15\% presentó TBC extra pulmonar. El 90\% de los casos de 2013 fueron casos nuevos y $24 \%$ presentó otro factor de riesgo, siendo los principales infección por $\mathrm{VIH}(7,4 \%)$, extranjero $(5,3 \%)$, diabético $(5,3 \%)$ y abuso de sustancias $(5,3 \%)$.

\section{Conclusiones}

La TBC en Chile continúa siendo un problema de salud pública relevante. El perfil epidemiológico actual muestra que la enfermedad se ha ido concentrando en los grupos de riesgo para desarrollar la enfermedad, especialmente en las poblaciones más jóvenes. Los principales factores de riesgo en Chile según los datos actuales son la co-infección por VIH, ser extranjero y la privación de libertad, cuyas tasas de incidencia superan ampliamente

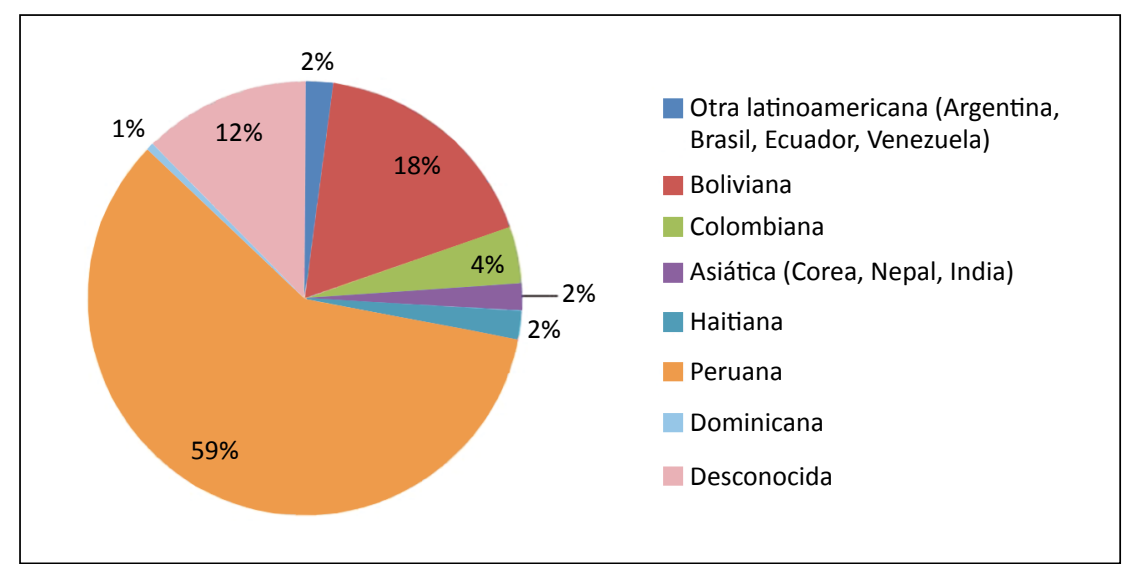

Figura 1. Distribución de casos de tuberculosis en extranjeros, según nacionalidad. Chile 2013. Fuente: Registro Nacional de Tuberculosis.

Tabla 2. Proporción de casos de tuberculosis en extranjeros respecto al total de casos en los Servicios de Salud seleccionados. Chile, 2013

\begin{tabular}{ll|} 
Servicio de Salud & $\%$ \\
Metropolitano Norte & 40 \\
Metropolitano Central & 31 \\
Iquique & 29 \\
Antofagasta & 27 \\
Arica-Parinacota & 14 \\
\hline Fuente: Registro Nacional de Tuberculosis. & \\
\hline
\end{tabular}

la tasa de la población general. Además, muchos de estos enfermos comparten varios de los factores de riesgo, mostrando que existe una población vulnerable en la cual se deben focalizar las acciones de pesquisa para permitir mejorar el diagnóstico precoz y con ello avanzar hacia la eliminación de la TBC en Chile.

\section{Resumen}

La tuberculosis continúa siendo un relevante problema mundial y nacional. En Chile, la tasa de incidencia se ha mantenido en 13 por cada 100.000 habitantes desde hace varios años sin la tendencia a la disminución esperada que permitiese su eliminación al año 2020. Como país de baja prevalencia, los casos se han ido concentrando en grupos de riesgo, llegando el año 2013 a observarse que el $33 \%$ de los casos pertenece a alguno de estos grupos, 
y esta proporción aumenta a medida que se analizan las personas más jóvenes. Los principales grupos de riesgo en Chile son la co-infección con VIH, los extranjeros y las personas privadas de libertad. Para el año 2013, la proporción de casos para estos tres grupos fue de $8,7 \%$,
$8,4 \%$ y $3,9 \%$ respectivamente, porcentajes que varían en forma importante al realizar el análisis regional. Además, muchos de estos enfermos presentan más de un factor de riesgo, lo que demuestra la existencia de grupos poblacionales más vulnerables a la tuberculosis.

\section{Referencias bibliográficas}

1.- World Health Organization. Global tuberculosis report 2014. 2014. http://www.who.int/tb/ publications/global_report/en/

2.- Programa Nacional de Control y Eliminación de la Tuberculosis. Tuberculosis: informe de situación Chile 2013 [Internet]. Santiago de Chile; 2014. Available from: http://web.minsal. cl/sites/default/files/files/TUBERCULOSISINFORME 2013.pdf

3.- Peña $C$, Farga $V$. El difícil camino del control sanitario de la tuberculosis. Rev Chil Enf Resp 2012; 28 (4): 311-8. Available from: http://www.scielo.cl/scielo.php?script=sci arttext\&pid=S0717-73482012000400008\&lng= es\&nrm=iso\&tlng=es

4.- Herrera T. Identificación y abordaje de la tuberculosis en poblaciones vulnerables: la experiencia de Chile. Reunión de países de baja prevalencia. Santiago de Chile: Programa Nacional de Control de la Tuberculosis; 2013. http://www.paho.org/hq/ index.php?option $=$ com_docman\&task $=\mathrm{doc}$ view\&gid=24866\&Itemid=

5.- Zúñiga M. La eliminación de la tuberculosis como problema de Salud Pública: Situación de Chile en el año 2008. Rev Chil Enf Resp 2009; 25 (2): 117-26. Available from: http://www.scielo.cl/scielo.php?script=sci arttext\&pid=S0717-73482009000200009\&lng= es\&nrm=iso\&tlng=es

6.- Ramonda P, Pino P, Valenzuela L. Diabetes mellitus como factor predictor de tuberculosis en el Servicio de Salud Metropolitano Sur en Santiago, Chile. Rev Chil Enf Resp 2012; 28 (4): 277-85. Available from: http://www.scielo. cl/scielo.php?script=sci_arttext\&pid=S0717$73482012000400003 \& \operatorname{lng}=$ es\&nrm=iso\&tlng= es

7.- García C. Tuberculosis en grupos de riesgo en la Región Metropolitana: 2008. Rev Chil Enf Resp 2010; 26 (2): 105-11. Available from: http://www.scielo.cl/scielo.php?script=sci arttext\&pid=S0717-73482010000200008\&lng= es\&nrm=iso\&tlng=es

8.- Programa Nacional de Control y Eliminación de la Tuberculosis. Normas técnicas para el control y la eliminación de la tuberculosis. 2014. Available from: http://web.minsal. cl/sites/default/files/NORMA_TECNICA_ TUBERCULOSIS.pdf

9.- Herrera T, Leiva E, Martin F, Miranda M, Morales C. Asociación entre tuberculosis y diabetes mellitus en la Región Metropolitana. Rev Chil Enf Resp 2013; 29: $171-5$

10.- Organización Mundial de la Salud. Global tuberculosis report 2012. 2013. http://www.who.int/tb/publications/global_ report/en/

11.- Villarroel L, Rabagliati R, Balcells M E, Karzulovic L, Pérez C. Tuberculosis en individuos con infección por VIH en Chile: Estudio de prevalencia e impacto sobre mortalidad. Rev Med Chile; 2008; 136 : 578-86.

12.- Organización Internacional para las Migraciones. Migración internacional, salud y derechos humanos. 2013. Available from: http://www.ohchr.org/ Documents/Issues/Migration/WHO_IOM_ UNOHCHRPublication_sp.pdf

13.- Universidad del Desarrollo. Investigadores UDD presentan estudio sobre la tuberculosis en cárceles chilenas [Internet]. Facultad de Medicina. 2014 [cited 2014 Dec 29]. Available from: http://medicina.udd.cl/noticias/2014/03/ investigadores-de-la-facultad-de-medicinapresentan-estudio-sobre-la-tuberculosis-encarceles-chilenas/ 